Thorax (1973), 28, 247.

\title{
A new instrument and technique for lung biopsy using local anaesthesia
}

\author{
D. T. THOMPSON \\ Department of Surgery, University of Rhodesia, Salisbury, Rhodesia
}

The more generally used techniques of lung biopsy are examined. There follows a description of a new instrument and technique for removing a small portion of lung using local anaesthesia. The technique, its advantages, and possible shortcomings are discussed.

The problem of crossing the pleural cavity to obtain a specimen of lung for examination without at the same time leaving a pneumothorax or causing haemorrhage has hindered the investigation of diffuse lung disease.

The various techniques used have been a formal, though limited, thoracotomy, needle biopsy, and, more recently, high-speed air-drill biopsy. Each of these techniques has complications; the open thoracotomy, although limited, is still a major procedure carrying risks and requiring stay in hospital. Needle biopsy often fails to obtain a satisfactory specimen and fatal haemorrhage has been reported. Drill biopsy, while obtaining a satisfactory specimen in $87 \%$ of cases, gives rise to pneumothoraces in $15 \%$ of patients and occasionally mild haemoptyses.

A problem not discussed concerning the use of drill biopsy is the fear engendered in nervous people, especially by the noise of the procedure. While this can be overcome with the more socially advanced patient by reassurance and perhaps some sedation, it is a matter of greater concern in a less sophisticated patient population.

The offer of a thoracotomy for lung biopsy to an African patient is invariably refused, fear of anaesthesia being the major reason for refusal. On the other hand, the high-speed drill technique is found to be very frightening. Another drawback is the cost and complexity involved in organizing the full apparatus required for the successful practice of the technique.

With these conditions in mind the following procedure for taking lung tissue for examination was evolved. It is now routinely used for obtaining specimens of lung and pleura for examination in this hospital.
The instrument (Fig. 1) consists of three parts : (1) a simple cannula with handle, the cannula of just sufficient length to allow the instrument to pass through an intercostal space to the pleura, and of a size to allow a good sample of lung to be taken and yet pass readily through an intercostal space; (2) a trocar with a central canal down its length which is continuous with an extension stem of the same diameter attached above; (3) a stilette fitting the central canal and having a coned faceted tip which gives with the trocar a flush, penetrating point.

\section{TECHNIQUE}

The patient is given a sedative as premedication and, after infiltration of local anaesthetic agent (usually lignocaine $1 \%$ ) in the appropriate intercostal space into muscle and down to pleura, a small $(3-5 \mathrm{~cm})$ incision is made and dissection is carried to the intercostal muscles, the fibres of which are separated. A small swab soaked in the lignocaine is then placed on the pleura for a few minutes. With the parts assembled, the trocar and cannula are pushed through the parietal pleura (Fig. 2), the patient being asked to blow up a balloon at this moment to maintain positive intrapleural pressure.

The trocar and stilette are removed and a segment of lung is delivered into the cannula with forceps as the patient continues to blow. With a number of patients, when the lung is free in the pleural cavity and the operation is being done over the lingular segment, the act of blowing herniates a portion of lung through the cannula (Fig. 3). This has not been noted in operations on the right side. As soon as the cannula is obstructed by lung, which is then held in that position, further blowing is unnecessary. A clamp is placed across the lung segment, the tip of which is excised and the cut edge stitched (Fig. 4), the 


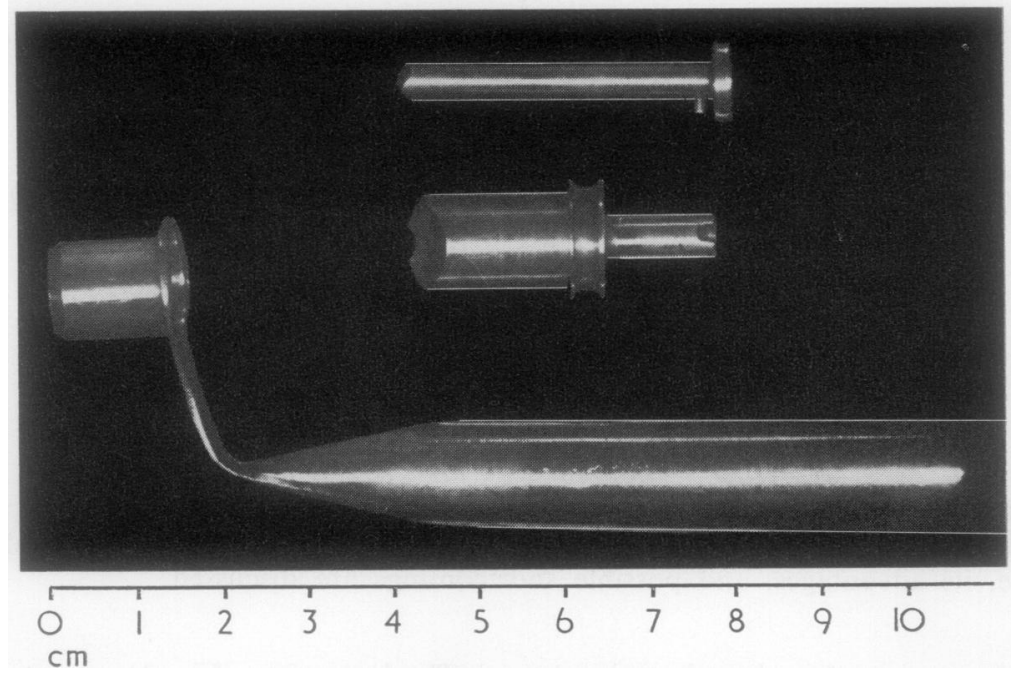

FIG. 1. Component parts of instrument.

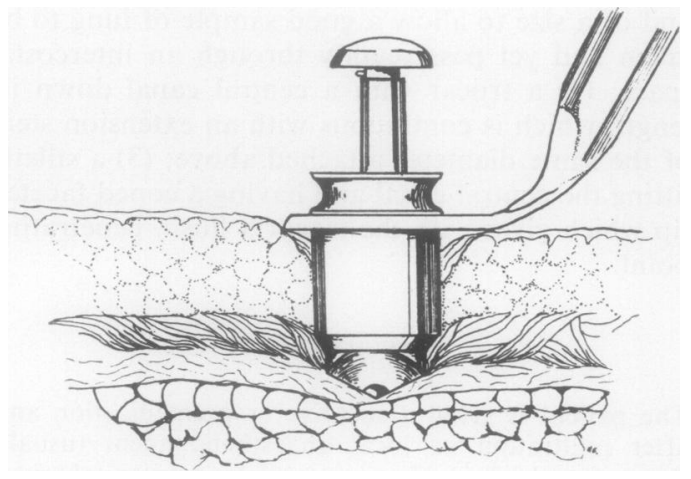

FIG. 2. Instrument in use just prior to penetration of the pleura.

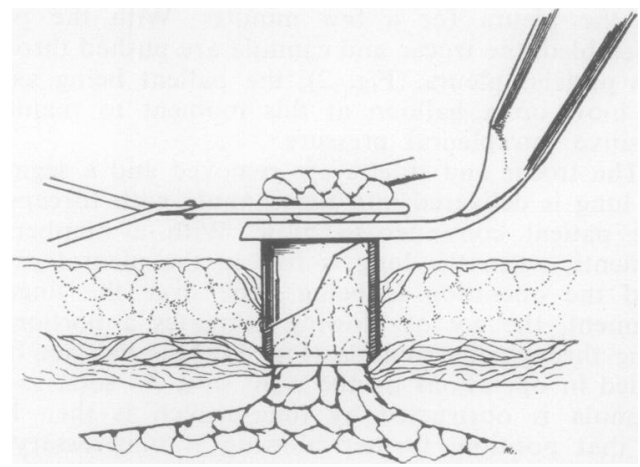

FIG. 3. Lung herniated through cannula and clamped.

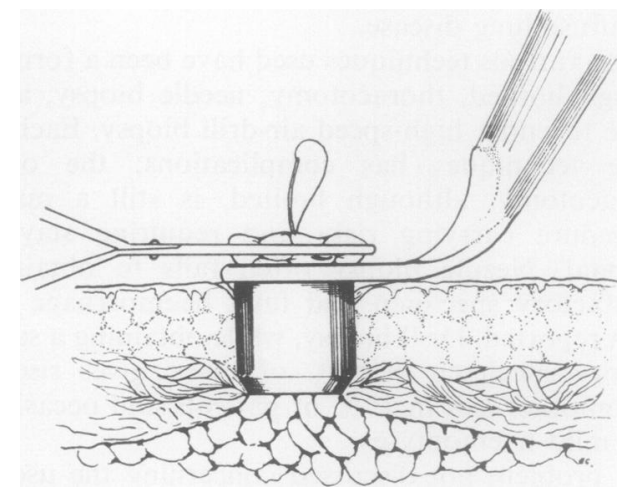

FIG. 4. Running stitch inserted proximal to clamp.

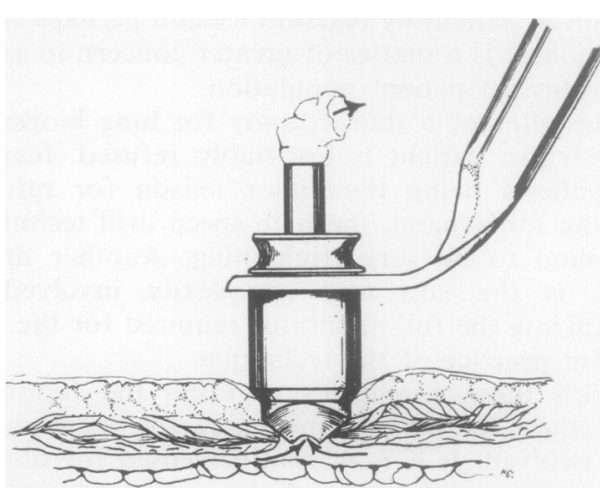

FIG. 5. Suction being applied to reinserted trocar. 
lung being held impacted in the cannula as the patient breathes normally.

The trocar without the stilette is now reintroduced and suction is applied to the stem (Fig. 5). Two stitches are inserted through the intercostal muscles on each side of the cannula and these are tied together as the instrument is removed. The incision is then closed and a small dressing is applied.

\section{DISCUSSION}

This technique has been used in 28 patients to investigate the fairly common, rather diffuse mottled lung fields on a chest film which, on mass radiography, had been diagnosed as miliary tuberculosis but without supporting evidence. It was felt, too, that a simple lung biopsy technique would help towards a more accurate diagnosis of bilharzial involvement of the lung, often suspected but not easy to prove. It is not anticipated that this technique would apply to solid lesions or to lesions in the apex of the lung - a possible disadvantage that would apply to present techniques other than open thoracotomy. It is rare, however, for biopsy to be done under these conditions. Since the introduction of this technique it has been easier to obtain permission for this investigation, and although the patients have been kept in hospital for observation and to await the report, in most cases they could have been discharged immediately.

It was thought at first that an adherent lung would prevent the taking of a specimen for biopsy, but the reverse has been the case. Having established that the lung is adherent, fear of a pneumothorax is eliminated, the trocar is removed, and a small segment of lung tissue is taken. Packing the wound with a damp hot swab has been sufficient to control bleeding and air leak. In two cases, as a precaution an intercostal tube was left in situ overnight, but no bleeding nor air leak occurred, and the tube was removed the following day.

In two patients, one an early case when the technique was being developed and the second treated by an assistant who thought he had achieved adequate closure, pneumothoraces were caused, the first barely visible on radiography, the second requiring intubation and under-water drainage for 24 hours.

Postoperative pain has been noted in a few cases, not severe, and controlled by codeine tablets. It has subsided in $\mathbf{4 8}$ to $\mathbf{7 2}$ hours.

In all patients good specimens have been obtained and no worrying complications arose, though one patient had inflammatory redness of the wound without suppuration. Advantages of the technique are its simplicity and minimal complications which can be reduced with increasing expertise.

Specimens of pleura also are easily and accurately obtained, and the surgeon in the act of taking a section of lung can report on the visual and tactile features of the lung. This, of course, would not apply to adherent lung. The patient, if he lives in the vicinity of the hospital, may go home the same day after a short period of observation. Postoperative chest films were taken in the first few cases. Now, in the interest of economy, if I am satisfied with the lung closure I have not continued to take these as a routine nor have I needed to revise this decision at a later stage.

The technique has the advantage that it requires no supplementary equipment such as electrical power points or pressure cylinders, and its easy portability would allow its use in surveys in district areas to investigate such problems as bilharziasis and industrial lung diseases, thus obviating the expense of the present practice of admitting patients to hospital. A survey of this nature is being considered at the moment.

It has been stated that among the criteria for lung sampling for biopsy a treatable disease should be considered in the differential diagnosis, a criterion that was previously included because of the inherent hazards of past techniques of lung biopsy. It is suggested that the present technique reduces lung biopsy to the pure diagnostic procedure it should be.

\section{BIBLIOGRAPHY}

Andrews, N. C., and Klassen, K. P. (1957). Eight years' experience with pulmonary biopsy. J. Amer. med. Ass., $164,1061$.

Deeley, T. J. (1960). Drill biopsy. Results with a high speed pneumatic drill. Acta Un. int. Cancr, 16, 338.

Ellis, F. (1947). Needle biopsy in the clinical diagnosis of tumours. Brit. J. Surg., 34, 240.

Krumholz, R. A., and Weg, J. G. (1966). Percutaneous needle biopsy of the lung. J. Amer. med. Ass., 195, 38.

Lauby, V. W., Burnett, W. E., Rosemond, G. P., and Tyson, R. R. (1965). Value and risk of biopsy of pulmonary lesions by needle aspiration. Twenty-one years' experience. J. thorac. cardiovasc. Surg., 49, 159.

Manfredi, F., Rosenbaum, D., and Behnke, R. H. (1963). Percutaneous needle biopsy of the lung in diffuse pulmonary diseases. Ann. intern. Med., 58, 773.

Scadding, J. G. (1970). Lung biopsy in the diagnosis of diffuse lung disease. Brit. med. J., 2, 557.

Smith, W. G. (1964). Needle biopsy of the lung, with special reference to diffuse lung disease and the use of a new needle. Thorax, 19, 68.

- (1964). Needle biopsy of the lung. Lancet, 2, 318.

Steel, S. J., and Winstanley, D. P. (1967). Preliminary communications-trephine biopsy for diffuse lung lesions. Brit. med. J., 3, 30. 